\title{
Surgery combined with photodynamic therapy in the treatment of disfiguring acne inversa: the efficacy of reconditioning
}

\author{
Wenbo Bu, Liming Huang, Xu Chen, Fang Fang
}

Jiangsu Key Laboratory of Molecular Biology for Skin Diseases and STIs, Institute of Dermatology, Chinese Academy of Medical Sciences \& Peking Union Medical College, Nanjing, China

Adv Dermatol Allergol 2019; XXXVI (2): 237-238

DOI: https://doi.org/10.5114/ada.2019.84600

A 16-year-old male suffering from repetitive papules, pustules, nodules and abscesses of the scalp, face, and neck for one year was admitted to our hospital. The lesions had gradually diffused and thickened on the scalp and face to produce the appearance of cutis verticis gyrata. An examination showed widely distributed scattered papules, nodules, pustules, abscesses, sinuses and scarring, with a trench-like appearance on the scalp, face, and neck. The patient was diagnosed with acne inversa with secondary cutis verticis gyrata. Corticosteroids, minocycline, and isotretinoin were orally administered, but the patient developed resistance to each drug. The patient had no other complicating disease, history of infection, or family genetic history.

A routine examination and an imaging examination after hospital admission showed no abnormalities. The pathology findings indicated neutrophil and lymphocyte infiltration in the full-thickness of the derma, with epithelial-like cells and foreign body giant cells. A microbial culture revealed Proteus mirabilis, Staphylococcus epidermidis and Klebsiella pneumoniae. After admission, multiple debridement drainage for the abscesses and sinuses of the scalp, face, and neck was performed, and then surgical removal of the excess superficial skin tissue was conducted, followed by suturing to correct the trench-like cranial skin. After removal of the scattered papules, pustules and abscesses as well as the skin lesions of the scalp, face, and neck, the wound surface was treated with photodynamic therapy. The patient's appearance was significantly improved, and no recurrence was observed during ten months of follow-up (Figure 1).
Cutis verticis gyrata-like acne inversa was rarely reported [1], and its treatment was difficult. In this case, the secondary trench-like cranial skin had formed due to the repetitive abscesses, sinuses, and severe scarring, leading to serious effects on the appearance of the head and face. The experience of this case suggests that the disfigurement caused by severe acne can be reconstituted through the excision of the scar lesions. Photodynamic therapy has been reported to improve acne lesions and reduce scar formation in the treatment of acne conglobata [2]. Actually, our case verified that photodynamic therapy could exhibit an assistant efficacy in combination with surgery for promoting the wound healing and improving acne lesions. Severe acne inversa is usually challenging to treat [3], particularly in patients who exhibit drug resistance. Our case suggests that surgery combined with photodynamic therapy could be considered as a preferred strategy for reconditioning the disfiguration due to acne inversa.

\section{Acknowledgments}

Wenbo Bu and Liming Huang contributed equally to this article.

Wenbo Bu and $\mathrm{Xu}$ Chen are supported by the PUMC Youth Fund and Fundamental Research Funds for the Central Universities (3332015116, 3332014008, 2016RC320005 and 2016ZX320014).

\section{Conflict of interest}

The authors declare no conflict of interest.

Address for correspondence: Xu Chen, Fang Fang, Jiangsu Key Laboratory of Molecular Biology for Skin Diseases and STIs, Institute of Dermatology, Chinese Academy of Medical Sciences \& Peking Union Medical College, Nanjing, China, 12 Jiangwangmiao St, 210042 Nanjing, China, e-mail: chenx@pumcderm.cams.cn, fangfangjh@126.com Received: 12.11 .2017 , accepted: 28.12.2017. 

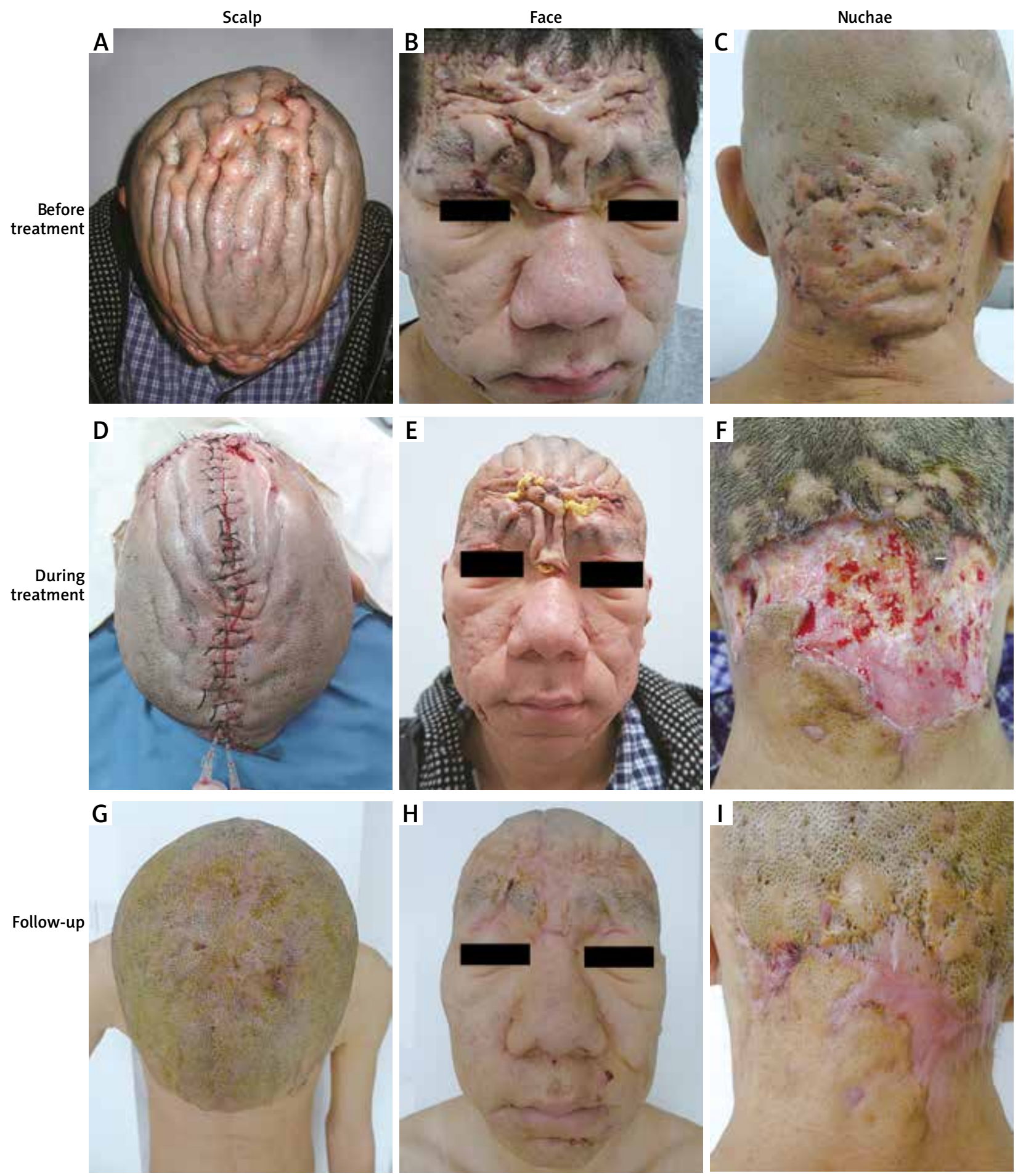

Figure 1. The clinical manifestation on the scalp (A), face (B) and nuchae (C). D-F - the suturing to correct the trench-like cranial skin, multiple debridement drainage for the abscesses and sinuses and surgical excision of the excess superficial skin tissue, respectively. G-I - present the significantly improved appearance after combined therapy

\section{References}

1. Lehmann P. Cutis verticis gyrata-like acne inversa. Combined medical and surgical management. Hautarzt 2009; 60: 328-30.

2. Yang GL, Zhao M, Wang JM, et al. Short-term clinical effects of photodynamic therapy with topical 5-aminolevulinic acid for facial acne conglobata: an open, prospective, parallelarm trial. Photodermatol Photoimmunol Photomed 2013: 29: 233-8.

3. Zouboulis CC, Desai N, Emtestam L, et al. European S1 guideline for the treatment of hidradenitis suppurativa/acne inversa. J Eur Acad Dermatol Venereol 2015; 29: 619-44. 